\title{
Pulmonary Fibrosis Due to Nitrofurantoin Therapy: A Case Report
}

\author{
Leonidas Grigorakos 1,2, Garyphallia Poulakou ${ }^{3}$, Daria Lazarescu2 ${ }^{2}$ Pavlos Myrianthefs ${ }^{1}$, \\ Nikolaos Markou², Maria Bikou², Adamantia Petineli", Konstantinos Kokkinis
}

${ }^{1}$ Faculty of Nursing, National and Kapodistrian University of Athens, Athens, Greece

${ }^{2}$ Intensive Care, "KAT" Trauma Hospital of Athens, Athens, Greece

${ }^{3}$ Fourth Department of Internal Medicine, Infectious Disease Unit, Attikon University General Hospital of Athens, Athens, Greece ${ }^{4}$ Radiology Department, "KAT" Trauma Hospital of Athens, Athens, Greece

Email: grigorakos@parliament.gr

How to cite this paper: Grigorakos, L., Poulakou, G., Lazarescu, D., Myrianthefs, P., Markou, N., Bikou, M., Petineli, A. and Kokkinis, K. (2017) Pulmonary Fibrosis Due to Nitrofurantoin Therapy: A Case Report. Open Journal of Respiratory Diseases, 7, 117124

https://doi.org/10.4236/ojrd.2017.73012

Received: July 17, 2017

Accepted: August 12, 2017

Published: August 15, 2017

Copyright $\odot 2017$ by authors and Scientific Research Publishing Inc. This work is licensed under the Creative Commons Attribution International License (CC BY 4.0).

http://creativecommons.org/licenses/by/4.0/

\begin{abstract}
We report the case of a patient with pulmonary fibrosis, developed as an adverse reaction to nitrofurantoin therapy received for totally 6 months for the prevention of recurrent urinary tract infections. Chest X-ray and CT scan revealed extensive elements of interstitial pulmonary fibrosis. After diagnosis, administration of nitrofurantoin was immediately stopped; and specific prolonged therapy with low-dose corticosteroids per os and inhaled steroids were administered. The patient responded successfully both clinically and biochemically and possible digestive system side effects were prevented through the administration of gastroprotection medication. For the prevention of urinary tract infection, the patient received well tolerated therapy with fosfomycin which was further continued as a prophylactic agent.
\end{abstract}

\section{Keywords}

Nitrofurantoin, Lung Toxicity, Fibrosis, Fosfomycin

\section{Introduction}

Prostatitis has always been considered as a difficult infection to treat due to limited penetration of many antimicrobial agents into the prostatic tissue. In order to bypass poor bioavailability, prolonged courses of antibacterial regimens are required that may extent to 12 weeks. Fluoroquinolones, once were the cornerstone of antimicrobial therapy for prostate infections, are nowadays frequently ineffective due to emergence of resistance. The high prevalence of multi-drug resistant organisms has negatively impacted the microbial epidemiology 
of prostatitis and thus has limited oral treatment options. Frequently, nowadays the therapeutic approach mandates prolonged parenteral courses that unfortunately are frequently associated with high relapse rates [1] [2]. Nitrofurantoin is one of the drugs used to prevent urinary tract infections (UTIs) but it is also known to be associated with adverse pulmonary reactions [3] [4] [5] [6] [7]. Even though its incidence is not frequent, nitrofurantoin may be associated with lung injury [8]. Here we present the case of a 79-year-old male who developed pulmonary fibrosis which was finally attributed to the use of nitrofurantoin.

\section{Case Presentation}

A 79-year-old white male with unremarkable medical history, presented to the infectious diseases outpatient department with a history of recurrent UTIs. The patient began suffering from urine incontinence fifteen years ago following transurethral prostatectomy for benign prostate hyperplasia. He underwent cystoscopy three years ago and since then he presented with recurrent episodes of acute pyelonephritis due to Escherichia coli. The strain exhibited resistance to fluoroquinolones and trimethoprim/sulfamethoxazole during his first assessment in our Infectious Diseases Outpatient Unit (IDOU), precluding use of these two antibiotic classes as prophylaxis options.

Despite the patient's history of transurethral prostatectomy, we could not exclude the possibility of recurrences stemming from the remaining prostate tissue, although an MRI of the pelvis during a febrile episode did not reveal any inflammatory changes in the residual prostatic tissue. Given the available in vitro susceptible options, the patient was treated with prophylactic nitrofurantoin 100 mg once daily for a period of six months.

After six months, he presented to our IDOU with symptoms of dyspnea on exertion, crackles and fatigue that had intensified over the past two months. Physical exam revealed stable vital sings with mild hypoxemia $\left(\mathrm{PaO}_{2} 77 \mathrm{mmHg}\right.$ and $\mathrm{SpO}_{2}$ 90\%). Presumptions that pulmonary intoxication may have occurred due to suppressive therapy with nitrofurantoin, led us to proceed to further pulmonary examination through chest radiography and computed tomography (CT).

The chest radiography (Figure 1) and CT scan (Figure 2) revealed elements of interstitial pulmonary fibrosis.

In particular, thickening of the interstitial tissue was observed, which was more pronounced in the middle and especially lower pulmonary area, locally accompanied by cystic bronchiectasis and honeycombing, without pleural effusion. As adverse reaction to nitrofurantoin was considered, its administration was immediately ceased. However, other conditions were also considered and a Gomori methenamine silver stain was performed which did not reveal the presence of any fungal organisms. When pulmonary function tests were performed, a restrictive pattern of lung function was revealed: forced vital capacity $(\mathrm{FVC})$ within $57 \%$ of the reference value $(3.53 \mathrm{~L})$, forced expiratory volume in 1 second/forced vital capacity (FEV1/FVC) ratio of $65 \%$ of the reference value 


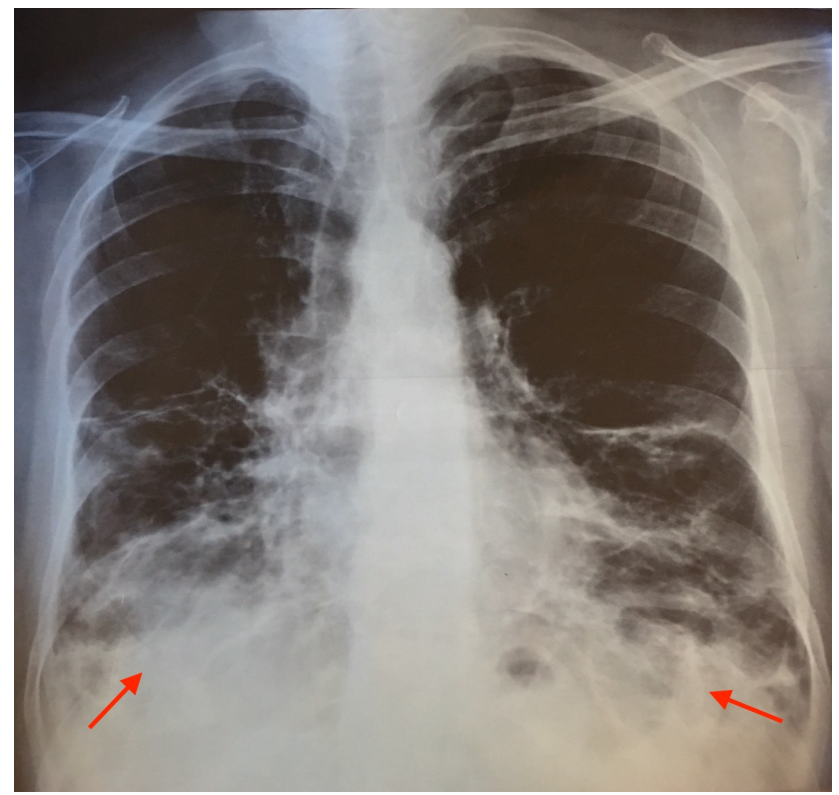

Figure 1. Chest radiography on admission showing extensive interstitial shadows in both lungs.

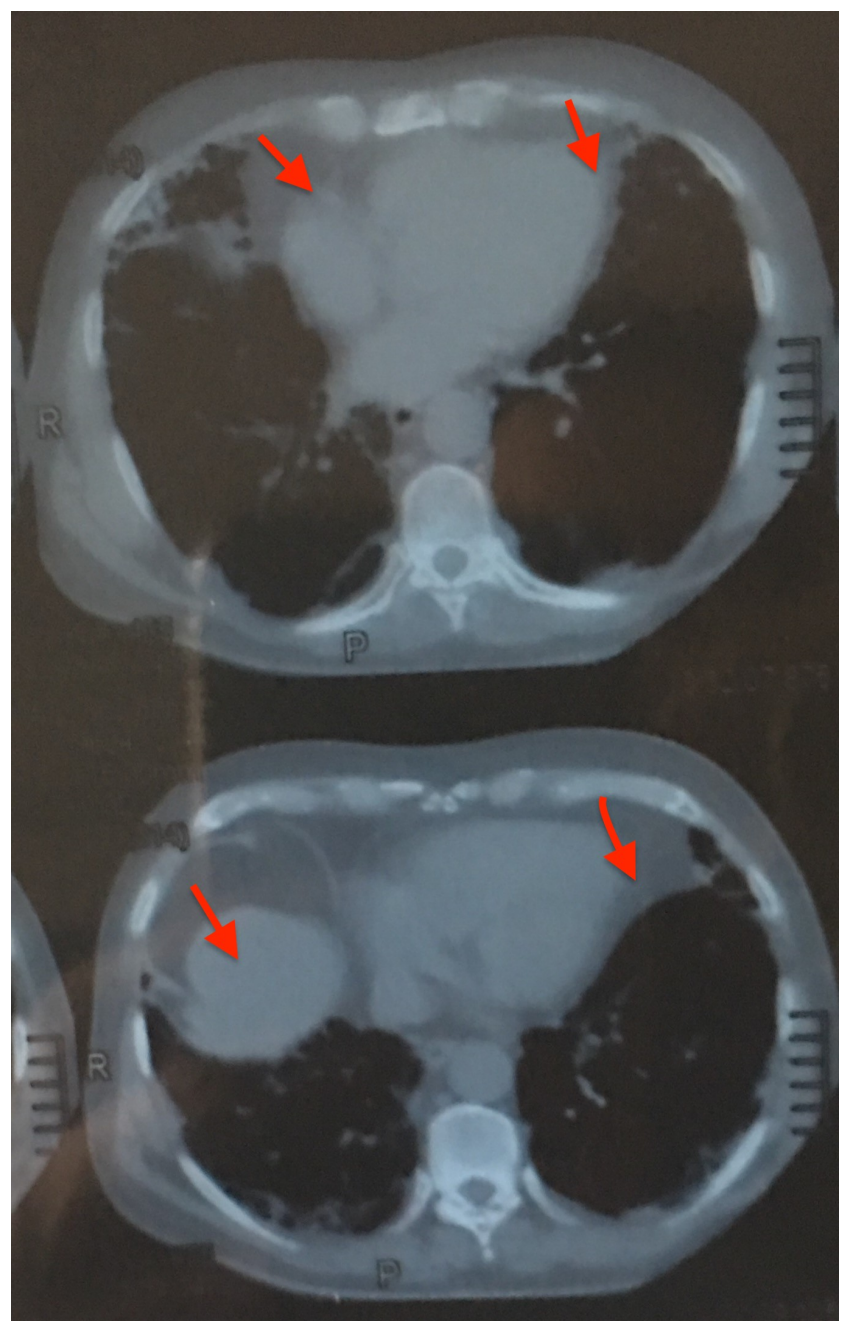

Figure 2. CT scan on admission showing extensive interstitial shadows in both lungs. 
(86\%), total lung capacity (TLC) was $70 \%$ of the one predicted $(6.38 \mathrm{~L})$ and the carbon monoxide diffusing capacity (DLCO) was $28 \%$ of the one predicted (21.2 $\mathrm{ml} / \mathrm{min} / \mathrm{mmHg}$ ).

As both pathologic findings and clinical course of nitrofurantoin long-term administration complied with chronic adverse drug reaction to this specific drug, our patient was counseled to stop its use. We immediately initiated corticosteroid therapy with methylprednisolone $(16 \mathrm{mg})$, which was administered per os (PO) for six months, initially at a loading dose of $48 \mathrm{mg} /$ day for one month. The dose was then tapered every one month by $8 \mathrm{mg} /$ day. After 6 -month treatment, administration of methylprednisolone was ceased. Supplementary, daily inhalations with fluticasone ( $125 \mathrm{mcg} /$ dose) three times per day were prescribed for twelve months. Corticosteroid therapy was well tolerated as we administered omeprozole $(20 \mathrm{mg} /$ day $)$ every morning in order to prevent injuries to the upper gastrointestinal tract.

Following the discontinuation of prophylactic nitrofurantoin the patient was placed on a prophylactic regimen with once daily trimethoprim/sulfamethoxazole $(800 / 160$ $\mathrm{mg}$ ). Follow-up urine culture one month later revealed more than $106 \mathrm{cfu} / \mathrm{ml} E$. coli, resistant to trimethoprim/sulfamethoxazole, nitrofurantoin and quinolones. Resistance profile precluded other orally administered agents, except for tetracyclines, therefore he received therapy with fosfomycin which was further continued as prophylactic agent.

Within one month, our patient observed some improvement of his dyspnea while after three months, his symptoms significantly diminished and the elements of interstitial pulmonary fibrosis which were previously found on his CT scan (Figure 3 ) and chest X-ray were significantly recessed (Figure 4).

Pulmonary function tests performed regularly afterward revealed both normalization of his FVC (growing to over $90 \%$ of predicted) within twelve months and progressive improvement in his DLCO. Thus, he could restart his usual activities without dyspnea or fatigue. After two years, a new CT scan (Figure 5) and chest radiography (Figure 6) revealed significant improvement of the radiographic image with partial regression of the lesions through the pulmonary network (interstitial fibrosis alterations).

During this period he was asymptomatic, with repeatedly sterile urine cultures and no signs of toxicity from laboratory follow up. During re-challenge, fosfomycin was well tolerated, with minor episodes of diarrhea treated with loperamide as needed.

\section{Discussion}

Pulmonary reaction secondary to nitrofurantoin is a potentially serious, even fatal, adverse drug reaction [9]. Both acute and chronic forms of nitrofurantoininduced pulmonary injury have been reported. The acute manifestation of this process is the most common and is thought to be due to a hypersensitivity reaction to the drug. Symptoms that develop after six months of therapy are generally considered to be chronic manifestation of the disease and have been thought 


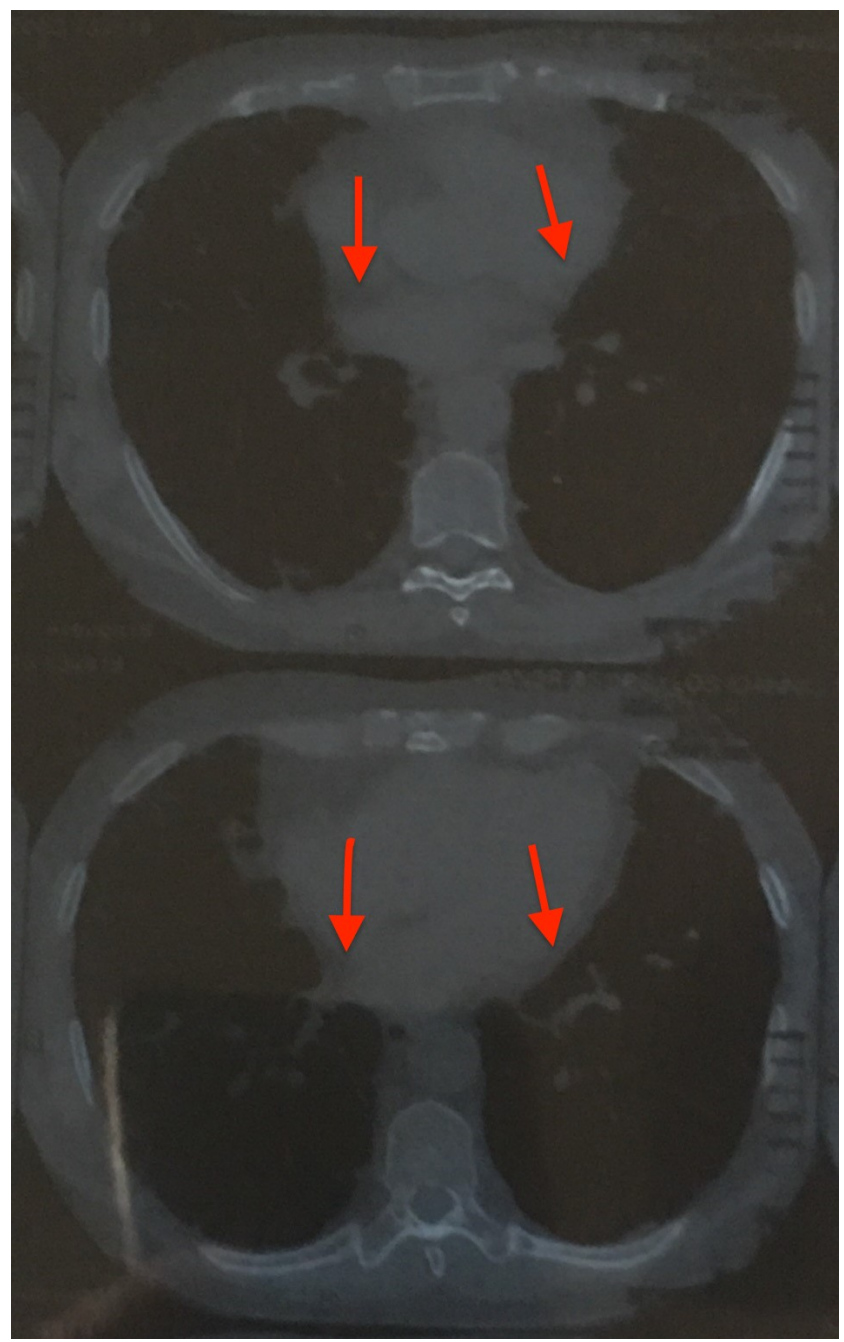

Figure 3. CT scan one month after treatment onset.

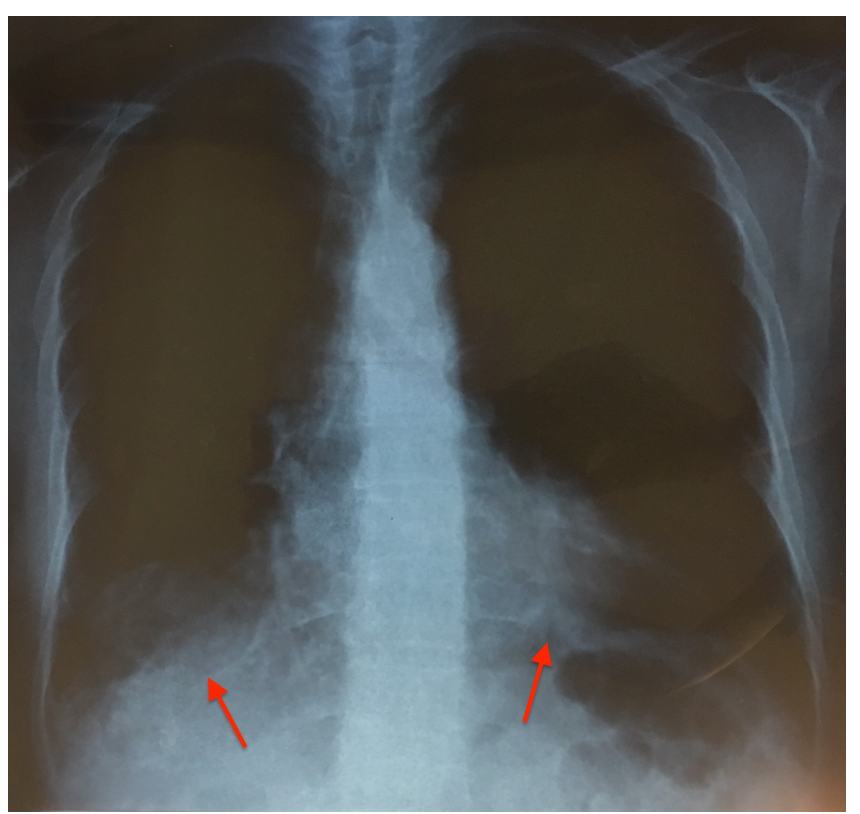

Figure 4. Chest radiography one month after treatment onset. 


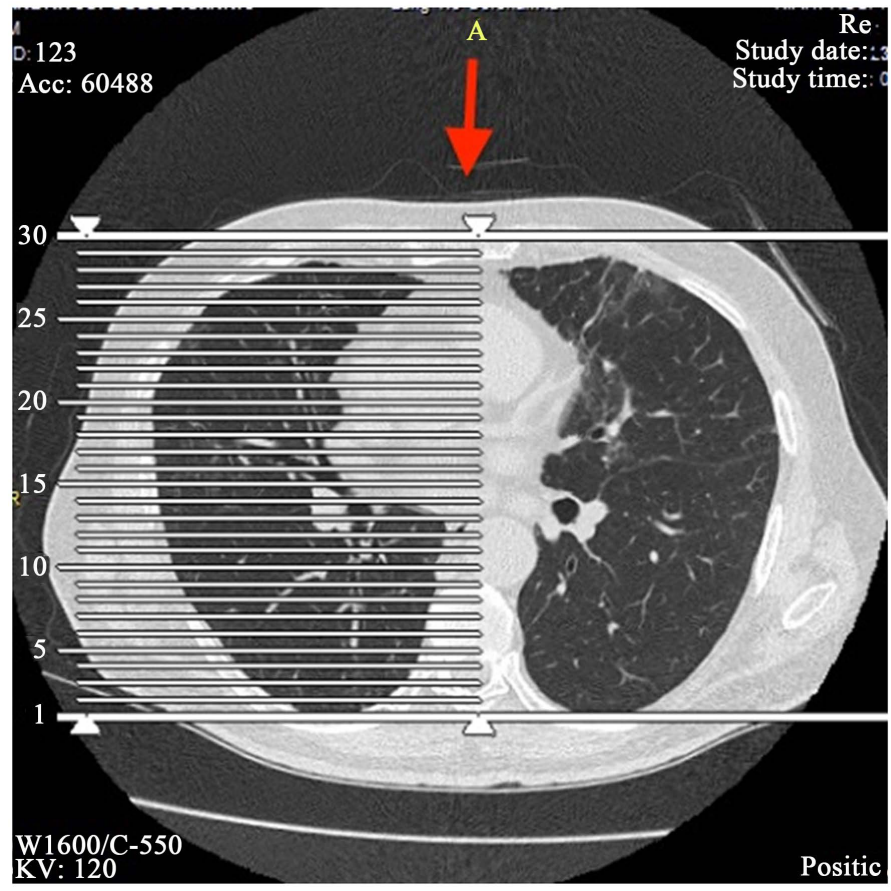

Figure 5. CT scan two years after the end of treatment.

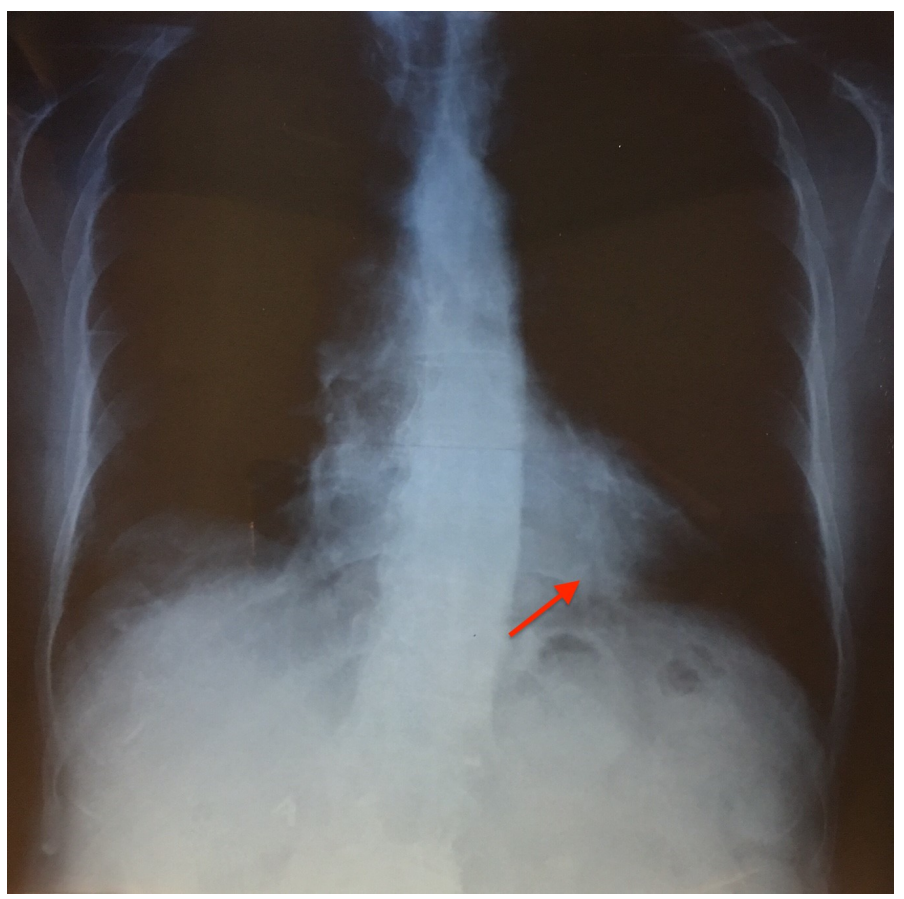

Figure 6. Chest radiography two years after the end of treatment.

to be the result of toxicity rather than hypersensitivity. Over the past decades, pulmonary reactions such as pulmonary fibrosis and bronchiolitis obliterans organizing pneumonia have been reported to be caused by exposure to nitrofurantoin [10]. Even though both timing and mechanisms of injury may be different, the treatment of chronic pulmonary injury from nitrofurantoin requires both drug interruption and therapy with corticosteroids [11] [12]. Recovery 
from chronic reactions may take from months to a year [13] [14] [15]. However, in chronic reactions, not all patients respond to drug abdication.

In our case, patient's advanced age and history of UTIs led us to decide the immediate cease of nitrofurantoin administration and to start orally a low-dose corticosteroid therapy, while a close monitoring was applied. For the treatment of UTIs, nitrofurantoin administration was replaced with fosfomycin, which has been proposed to have a potential role in prophylaxis or treatment of prostatitis without any report of toxicity issues regarding its use [16] [17].

Within the first three months, the patient experienced relative improvement of his physical condition and serial pulmonary function tests documented reversal of the restrictive pattern. Our experience and other recent case descriptions [13] [14] suggest that the older classification of nitrofurantoin lung toxicity as either acute or chronic, with the latter frequently irreversible is incomplete. Nevertheless, it remains very important to suspect a drug reaction whenever a patient taking nitrofurantoin develops respiratory symptoms. On the contrary, fosfomycin long-term administration was proven to be efficacious and well tolerated in terms of toxicity and should be considered as an alternative agent, especially in patients with paucity of other prophylaxis options due to resistance profile or adverse events such as pulmonary fibrosis.

\section{Conclusion}

To conclude, we underline that in case of documented pulmonary fibrosis and concurrent nitrofurantoin administration; both nitrofurantoins should be immediately stopped, and specific prolonged therapy with low-dose corticosteroids and inhaled steroids should be started. In parallel, appropriate clinical and laboratory monitoring should be applied until the resolution of symptoms and improvement of lab tests results. Additionally, administration of gastroprotection medication effectively prevents the possible adverse effects on the digestive system due to corticosteroids' use.

\section{Consent for Publication}

All procedures followed were in accordance with the ethical standards of the responsible committee on human experimentation (institutional and national) and with the Declaration of Helsinki 1975, as revised in 2000. Written informed consent was obtained from the patient for publication of this case report and any accompanying images. A copy of the written consent is available for review by the Editor-in-Chief of this journal.

\section{References}

[1] Goemaere, N., Grijm, K., van Hal, P. and den Bakker, M. (2008) Nitrofurantoin-Induced Pulmonary Fibrosis: A Case Report. Journal of Medical Case Reports, 2, 169. https://doi.org/10.1186/1752-1947-2-169

[2] Travis, W.D., Colby, T.V., Koss, M.N., Rosado-de-Christenson, M.L., Muller, N.L. and King, T.E. (2002) Non-Neoplastic Disorders of the Lower Respiratory Tract. In: Atlas of Nontumor Pathology, Vol. 2, The American Registry of Pathology, Wash- 
ington DC, 327.

[3] Bhullar, S., Lele, S.M. and Kraman, S. (2007) Severe Nitrofurantoin Lung Disease Resolving without the Use of Steroids. Journal of Postgraduate Medicine, 53, 111113. https://doi.org/10.4103/0022-3859.32211

[4] Muller, A.E., Verhaegh, E.M., Harbarth, S., Mouton, J.W. and Huttner, A. (2017) Nitrofurantoin's Efficacy and Safety as Prophylaxis for Urinary Tract Infections: A Systematic Review of the Literature and Meta-Analysis of Controlled Trials. Clinical Microbiology and Infection, 23, 355-362.

[5] Mendez, J., Nadrous, H., Hartman, T. and Ryu, J. (2005) Chronic NitrofurantoinInduced Lung Disease. Mayo Clinic Proceedings, 80, 1298-1302. https://doi.org/10.4065/80.10.1298

[6] Ali, S. and Baj, M. (2012) Is He Ill Because of His Pill? A Case Report of Nitrofurantoin-Induced Lung Injury. West of England Medical Journal, 111, 1.

[7] Koulaouzidis, A., Bhat, S., Moschos, J., Tan, C. and De Ramon, A. (2007) Nitrofurantoin-Induced Lung and Hepatotoxicity. Annals of Hepatology, 6, 119-121.

[8] Erasmus, J.J., Mc Adams, H.P. and Rossi, S.E. (2002) Drug-Induced Lung Injury. Seminars in Roentgenology, 37, 72-81. https://doi.org/10.1053/sroe.2002.0000

[9] Kanji, Z., Su, V. and Mainra, R. (2011) Nitrofurantoin-Induced Pulmonary Reaction Involving Respiratory Symptoms: Case Report. CJHP, 64, 362-365.

[10] Epler, G.R. (2004) Drug-Induced Bronchiolitis Obliterans Organizing Pneumonia. Clinics in Chest Medicine, 25, 89-94.

[11] Nishiyama, O., Shimizu, M., Ito, Y., Kume, H., Suzuki, R., Yokoi, T. and Yamaki, K. (2001) Effect of Prolonged Low-Dose Methylprednisolone Therapy in Acute Exacerbation of Idiopathic Pulmonary Fibrosis. Respiratory Care, 46, 698-701.

[12] Nicolle, L.E. (2005) AMMI Canada Guidelines Committee Complicated Urinary Tract Infection in Adults. Canadian Journal of Infectious Diseases and Medical Microbiology, 16, 349-360. https://doi.org/10.1155/2005/385768

[13] Weir, M. and Daly, G.J. (2013) Lung Toxicity and Nitrofurantoin: The Tip of the Iceberg? QJM, 106, 271-272. https://doi.org/10.1093/qjmed/hcs129

[14] Rego, L., Glazer, C. and Zimmern, P. (2016) Risks of Long-Term Use of Nitrofurantoin for Urinary Tract Prophylaxis in the Older Patient. Urological Science, 27, 193-198.

[15] Hardak, E., Berger, G., Krivoy, N., Solomonov, A. and Yigla, M. (2010) Nitrofurantoin Pulmonary Toxicity: Neglected Threat. Current Drug Safety, 5, 125-128.

[16] Gardiner, B.J., Mahony, A.A., Ellis, A.G., Lawrentschuk, N., Bolton, D.M., Zeglinski, P.T., et al. (2014) Is Fosfomycin a Potential Treatment Alternative for Multidrug-Resistant Gram-Negative Prostatitis? Clinical Infectious Diseases, 58. https://doi.org/10.1093/cid/cit704

[17] Grayson, M.L., Macesic, N., Trevillyan, J., Ellis, A.G., Zeglinski, P.T., Hewitt, N.H., et al. (2015) Fosfomycin for Treatment of Prostatitis: New Tricks for Old Dogs. Clinical Infectious Diseases, 61, 1141-1143. https://doi.org/10.1093/cid/civ436 
Submit or recommend next manuscript to SCIRP and we will provide best service for you:

Accepting pre-submission inquiries through Email, Facebook, LinkedIn, Twitter, etc. A wide selection of journals (inclusive of 9 subjects, more than 200 journals)

Providing 24-hour high-quality service

User-friendly online submission system

Fair and swift peer-review system

Efficient typesetting and proofreading procedure

Display of the result of downloads and visits, as well as the number of cited articles Maximum dissemination of your research work

Submit your manuscript at: http://papersubmission.scirp.org/

Or contact ojrd@scirp.org 